\title{
Effects of phased joint intervention on IL-35 and IL-17 expression levels in patients with portal hypertension
}

\author{
YUGANG WANG, JINBIN DONG, WENYING MENG, JIALI MA, NA WANG, JUE WEI and MIN SHI \\ Department of Gastroenterology, Shanghai Changning Central Hospital, Shanghai 200336, P.R. China
}

Received October 1, 2013; Accepted February 5, 2014

DOI: $10.3892 /$ ijmm.2014.1662

\begin{abstract}
The aim of the present study was to investigate the clinical efficacy of phased joint intervention [percutaneous transhepatic variceal embolization (PTVE) + phased partial splenic embolization (PSE)] in patients with portal hypertension complicated by esophageal variceal bleeding and hypersplenism and the effect of this intervention on interleukin-35 (IL-35)/IL-17 expression. A review of 53 patients with portal hypertension caused by liver cirrhosis and complicated by esophageal variceal bleeding and hypersplenism treated with phased joint intervention was conducted, and portal hemodynamics, routine blood examinations and liver function were determined. Quantitative polymerase chain reaction (qPCR) was used to evaluate EBI3, FOXP3 and IL-17 mRNA expression levels in peripheral blood mononuclear cells (PBMC) before and after the phased joint intervention, while western blot analysis was used to determine their protein expression. All 53 patients required emergency hemostasis resulting in an emergency hemostatic rate of $100 \%$. Varicose veins disappeared, portal hemodynamics and liver function improved subsequent to the intervention. The expression levels of EBI3, FOXP3 and IL-17 mRNA in the postoperative group were significantly lower than the preoperative levels $(\mathrm{P}<0.01)$. The protein expression levels of EBI3, FOXP3 and IL-17 in the postoperative group were reduced compared with the preoperative levels. The concentrations of IL-35, IL-6 and IL-17 in peripheral blood were significantly reduced after the phased joint intervention $(\mathrm{P}<0.01)$. Serum IL-35, IL-6 and IL-17 levels were positively correlated with total bilirubin and international normalized ratio, and negatively correlated with albumin. The phased joint intervention can effectively treat
\end{abstract}

Correspondence to: Dr Min Shi, Department of Gastroenterology, Shanghai Changning Central Hospital, 1111 Xianxia Street, Shanghai 200336, P.R. China

E-mail: shimingdyx@yeah.net

Abbreviations: PTVE, percutaneous transhepatic variceal embolization; PSE, partial splenic embolization; PBMC, peripheral blood mononuclear cells

Key words: esophageal varices, embolization, cirrhosis, interleukin-35, interleukin-17 esophageal variceal bleeding and hypersplenism, and improve liver function. The efficacy of this intervention may be associated with the regulation of immune function.

\section{Introduction}

Patients with cirrhosis usually have associated portal hypertension. Esophageal variceal bleeding (EVB) is one of the most common and dangerous complications of cirrhosis associated with portal hypertension (1). The majority of patients have acute onset of this disease with a large amount of bleeding which is difficult to stop leading to critical conditions and high mortality (2). Almost half of cirrhotic patients are diagnosed with esophageal varices, and those with liver function classified as Child-Pugh Class $\mathrm{B}$ or $\mathrm{C}$ are prone to a higher incidence of esophageal varices (3). Approximately $7 \%$ of patients develop esophageal varices each year $(4,5)$. The rate of initial hemorrhage is $\sim 12 \%$ in the first year (6), the recurrence rate of hemorrhage in 1 year is $\sim 60 \%$ (7) and mortality within 6 weeks of bleeding remains high at up to $20 \%$ (8).

Percutaneous transhepatic variceal embolization (PTVE) has been used since 1974 for the effective treatment of EVB (9). However, its clinical application is limited due to a high re-bleeding rate (10-12). Transcatheter splenic arterial embolization can decrease portal vein blood flow and pressure, and improve hypersplenic symptoms. However, a disadvantage of this technique is that $73 \%$ of patients have severe complications if the embolic volume is $>70 \%$ of the vein (13). We have used the improved phased joint intervention [PTVE + phased partial splenic embolization (PSE)] to treat portal hypertension complicated by EVB and hypersplenism since 2006, which avoids the weaknesses of other therapeutic methods, thus achieving satisfactory clinical efficacy (14). This study assessed the clinical application of phased joint intervention by reviewing patient data. However, the internal mechanism of this therapeutic strategy is not yet clear.

Cirrhosis is associated with varying degrees of splenomegaly and chronic liver disease, with the degree of splenomegaly being positively correlated with the degree of liver fibrosis. Immune regulation is one of the most important functions of the spleen, therefore partial hemisection of the spleen is conducive to the regeneration of liver cells. The initiation and promotion of inflammation is an important factor in the progression of liver fibrosis (15). Studies have shown that the immune system plays an important role in the formation of 
cirrhosis. Hepatic stellate cell (HSC) activation is a key process in liver fibrosis, and Kupffer cells (KC) (16), blood-derived monocytes/macrophages (17), natural killer (NK) cells (18), NK T cells (NKT) (18) and T cells $(19,20)$ synthesize and secrete cytokines, chemokines and other chemical media that affect the HSC-cytokine-chemokine immune microenvironment. The interplay of various factors in this environment forms a complex network system, affecting the process of liver fibrosis. The proinflammatory cytokine, interleukin-17 (IL-17), and the anti-inflammatory cytokine, IL-35, have become an important research topic in the evaluation of liver fibrosis.

This study investigated the protein and gene expression levels of IL-35 and IL-17 before and after interventional embolization, and determined the possible immune mechanisms of interventional embolization which affect the progression of liver cirrhosis.

\section{Materials and methods}

Patient characteristics. This study retrospectively analyzed 53 cases of liver cirrhosis complicated by portal hypertension, esophageal variceal bleeding and hypersplenism in our hospital from October 2006 to December 2011. Portal hypertension was mainly due to hepatic, autoimmune and alcoholic cirrhosis, and phased joint intervention (PTVE + phased PSE) was selected as the preferred treatment method.

In total, 53 patients, 37 males and 16 females with an average age of $47.92 \pm 8.00$ years (range, 44-71) were included. Liver function in these patients was classified as Child-Pugh class A in 15 cases, class B in 22 cases, and class $C$ in 16 cases. All 53 patients had a history of $\geq 1$ bleeding episodes and all 53 patients had received emergency interventional treatment including 8 cases with ascites (Table I).

Following approval by the Shanghai Changning District Central Hospital Ethics Committee, all patients agreed to this intervention, and the patients or their authorized family members signed written informed consent.

Inclusion and exclusion criteria. Preoperative examinations included routine blood examination, liver and kidney function, hepatitis virus markers, coagulation, abdominal ultrasound, endoscopy and computed tomography (CT). Inclusion criteria were as follows: cirrhotic portal hypertension diagnosed by clinical and imaging examinations; a history of $>1$ gastrointestinal bleeding episodes; esophageal varices diagnosed by endoscopy in the absence of other possible diseases resulting in upper gastrointestinal hemorrhage; and obvious hypersplenism. Exclusion criteria were: large ascites; combination of hepatocellular carcinoma, hepatorenal syndrome or spontaneous peritonitis; stage III-IV hepatic encephalopathy; portal vein thrombosis; severe cavernous transformation of portal vein; superselective failure caused by gastric coronary vein variation; cardiopulmonary dysfunction; total bilirubin $\geq 85 \mu \mathrm{mol} / 1$; and prolonged prothrombin time $(\mathrm{PT}) \geq 6 \mathrm{sec}$.

Materials. FTC-3000 real-time polymerase chain reaction (PCR) kit was purchased from Funglyn Biotech Inc., Scarborough, ON, Canada. SYBR-Green real-time PCR Master Mix (code \#QPK-201) was obtained from Toyobo Bio-Technology, Co., Ltd., China. IL-35 (EBI3), IL-17 and
Table I. Clinical features

\begin{tabular}{lc}
\hline Clinical features & Values \\
\hline No. of cases & 53 \\
Gender & \\
Male & 37 \\
Female & 16 \\
Age (years) & $47.92 \pm 8.00$ \\
Liver function Child-Pugh & \\
A & 15 \\
B & 22 \\
C & 16 \\
Cirrhosis etiology & \\
Hepatic cirrhosis & 35 \\
Autoimmune cirrhosis & 10 \\
Alcoholic cirrhosis & 8 \\
History of esophageal variceal bleeding & 53 \\
Hypersplenism & 53 \\
\hline
\end{tabular}

Table II. Primer probe sequences.

\begin{tabular}{|c|c|c|}
\hline mRNA & Sequence $\left(5^{\prime}-3^{\prime}\right)$ & $\begin{array}{l}\text { Amplicon } \\
\text { size (bp) }\end{array}$ \\
\hline $\begin{array}{l}\mathrm{IL}-35 \\
(\mathrm{EBI} 3)\end{array}$ & $\begin{array}{l}\text { F: TCATTGCCACGTACAGGCTC } \\
\text { R: GGGTCGGGCTTGATGATGTG }\end{array}$ & 208 \\
\hline IL-17 & $\begin{array}{l}\text { F: AGATTACTACAACCGATCCACCT } \\
\text { R: GGGGACAGAGTTCATGTGGTA }\end{array}$ & 151 \\
\hline FOXP3 & $\begin{array}{l}\text { F: GTGGCCCGGATGTGAGAAG } \\
\text { R: GGAGCCCTTGTCGGATGATG }\end{array}$ & 238 \\
\hline$\beta$-actin & $\begin{array}{l}\text { F: TGGAGAAAATCTGGCACCA } \\
\text { R: CAGGCGTACAGGGATAGCAC }\end{array}$ & 189 \\
\hline
\end{tabular}

F, forward; R, reverse; IL, interleukin.

FOXP3 primers were designed in our laboratory and synthesized by Shanghai Generay (Shanghai, China) (Table II).

The following antibodies were used: Rabbit anti-IL-17 polyclonal antibody (Abcam, cat. \#ab79056); mouse anti-IL-35 monoclonal antibody (R\&D Systems, cat. \#MAB1570); rabbit anti-FoxP3 (D25D4) monoclonal antibody (CST, cat. \#5298); mouse anti- $\beta$-actin monoclonal antibody (Sigma, cat. \#A5316); goat anti-rabbit IgG (whole molecule)-peroxidase antibody (Sigma, cat. \#A0545); goat anti-mouse IgG (Fc specific)-peroxidase antibody (Sigma, cat. \#A0168); BCA kit: Pierce BCA Protein assay kit (Thermo Scientific, cat. \#23227); ECL reagent: Amersham ECL plus Western Blot Detection system (GE Healthcare; San Diego, CA, USA) (cat.\#RPN2132); enzymelinked immunosorbent assay (ELISA) kit (Uscn Life Science Wuhan, China), the GE3100 flat C-DSA system was purchased from GE Healthcare; and Color Doppler ultrasound diagnostic equipment GE730 was purchased from GE Healthcare. 
Phased joint embolization. Phased joint intervention (PTVE and PSE) was utilized for which the PSE embolization range was controlled between 30 and 40\%. PSE was conducted again 3 months later with the embolization range controlled between 30 and $40 \%$.

Percutaneous transhepatic variceal embolization. The right portal vein was punctured percutaneously and transhepatically with a $21 \mathrm{G}$ needle (Cook, Bloomington, IN, USA) after the liver area was routinely sterilized and positioned. After successful puncture, a guide wire was inserted into the superior mesenteric vein along the needle, and then a $5 \mathrm{~F}$ vascular sheath (Cook) was inserted along the guide wire. The contrast agent was injected for splenic and portal vein angiography, respectively, under digital subtraction angiography (DSA) perspective conditions. The catheter tip was then superselectively inserted into the stomach coronary vein to image esophageal varices. According to DSA development, 5-30 $\mathrm{ml}$ of absolute ethyl alcohol were injected for the distal embolization of the gastric coronary vein. In the case of slow blood flow, a spring ring of the appropriate specifications (Cook) was used for main vein thrombosis. When the angiographic image showed complete occlusion of the gastric coronary vein, short gastric vein thrombosis was conducted using the same method. In the case of fast blood flow, a steel ring of 5-10 mm diameter was applied firstly for embolization to slow the blood flow of obviously thickened varicose branches. In addition, some patients required gelatin sponge particles. Absolute ethyl alcohol was then injected slowly until the varicose vein was no longer visible. DSA contrast was administered again to determine the effect of embolization. The catheter tip was inserted into the splenic vein again to determine the presence of varicose veins. If varicose veins were observed, the same method was applied for complete embolization. After treatment, the catheter sheath was slowly removed from the right portal vein to ensure no bleeding. In the case of bleeding, a gelatin sponge was used to block the needle with a pressure dressing to ensure no bleeding for $48 \mathrm{~h}$.

Partial splenic embolization. The catheter was inserted using the Seldinger method via the femoral artery to reach the splenic hilum, the medium and lower branch through the proximal splenic artery. DSA was used to identify the splenic vessels and blood flow, gelatin sponge particles were injected into the splenic artery and its branches for embolization, splenic arteriography was conducted, and a pressure dressing was placed on the puncture point after the catheter was removed.

Postoperative treatment. Vital signs and abdominal conditions were closely observed. Patients who received pressure dressings and compression hemostasis were required to have a 24-h bed rest and measures were taken to prevent infection and other complications.

Color doppler ultrasound hemodynamics. B-type ultrasonic color Doppler diagnostic apparatus with a probe frequency of 3.5 $\mathrm{MHz}$ was operated by appointed personnel from the ultrasound department to determine the diameter of the portal vein (DPV), diameter of the splenic vein (DSV), maximum flow velocity (Vmax) and average flow velocity of veins $(\mathrm{V})$. Blood flow was calculated using the formula, $Q=(D / 2)^{2} \times \pi \times 0.57 \times$
Vmax x 60, as described in the study by Moriyasu et al, when patients were in a quiet inspiratory breath-hold position (21). These parameters were measured three times to obtain a mean value, and each result was double-blinded.

Assessment of efficacy. Patients underwent regular re-examinations of routine blood measurements, liver function, gastroscopy, color Doppler ultrasound and CT. The 6-month follow-up period included examinations of routine blood test, liver function, complications, and re-bleeding. In the event of gastrointestinal bleeding, patients were seen by a doctor immediately. Postoperative hemodynamics of the portal vein system was evaluated using abdominal ultrasound. Postoperative indicators of liver function included alanine transaminase (ALT), albumin (ALB), total bilirubin (TBIL) and the PT/international normalized ratio (INR), while indicators of hypersplenism improvement included white blood cell (WBC) and platelet (PLT) counts. The above indicators were determined prior to the intervention and 1,4 (one month after phased PSE) and 6 months after surgery. In addition, temperature changes, abdominal pain, pleural effusion, peritonitis, splenic abscess and other adverse reactions were assessed. The serum levels of IL-35, IL-6 and IL-17 were analyzed by ELISA according to the manufacturer's instructions.

Isolation, cryopreservation and resuscitation of peripheral blood mononuclear cells (PBMC). When patients visited the doctor at follow up, a $5 \mathrm{ml}$ sample of fresh blood was collected each time [before surgery and 4 (one month after phased PSE) months after surgery], placed in an ethylenediaminetetraacetic acid (EDTA) anticoagulant tube, and sent to the laboratory within 2 h. Peripheral blood samples from 32 healthy subjects were collected and used as controls, and informed consent forms were signed. The samples were centrifuged at $400 \mathrm{x} \mathrm{g}$ for 5 min to obtain the plasma which was stored at $-70^{\circ} \mathrm{C}$. The remaining cell pellets were mixed with $3 \mathrm{ml}$ phosphate-buffered saline (PBS) solution, added slowly to $3 \mathrm{ml}$ of prepared Ficoll lymphocyte separation medium along the pipette wall, and transferred to a horizontal rotor at $800 \mathrm{x} \mathrm{g}$ for $15 \mathrm{~min}$. Mononuclear cells in the intermediate liquid layer were added to a Falcon tube containing $3 \mathrm{ml}$ PBS solution. The mononuclear cells were washed at $200 \mathrm{x} \mathrm{g}$ for $10 \mathrm{~min}, 5 \mathrm{ml}$ of PBS solution was added after the supernatant was removed, washed once at $200 \mathrm{x} \mathrm{g}$ for $10 \mathrm{~min}$, counted using a microscope and preserved after the supernatant was removed. The cells in three tubes were frozen in $500 \mu 1$ RPMI-1640 culture medium containing 10\% dimethyl sulfoxide (DMSO) and 10\% fetal bovine serum (FBS), placed in the freezer at $-80^{\circ} \mathrm{C}$ overnight, and stored in liquid nitrogen the following day.

Quantitative PCR. Total RNA was extracted using TRIzol and RNA purity and concentration were determined using a UV spectrophotometer. Total RNA ( $2 \mu \mathrm{g}$ ) was reverse transcribed into cDNA. Real-time fluorescence was used to quantify PCR with the Toyobo ReverTra Ace qPCR RT kit system. RT products were amplified for $\beta$-actin, EBI3, FOXP3 and IL-17 genes. The mRNA expression and gradation in each group were compared with a fully automatic gel imaging analysis system after the amplified products were subjected to gel electrophoresis. Results were corrected using $\beta$-actin. Table I shows the 

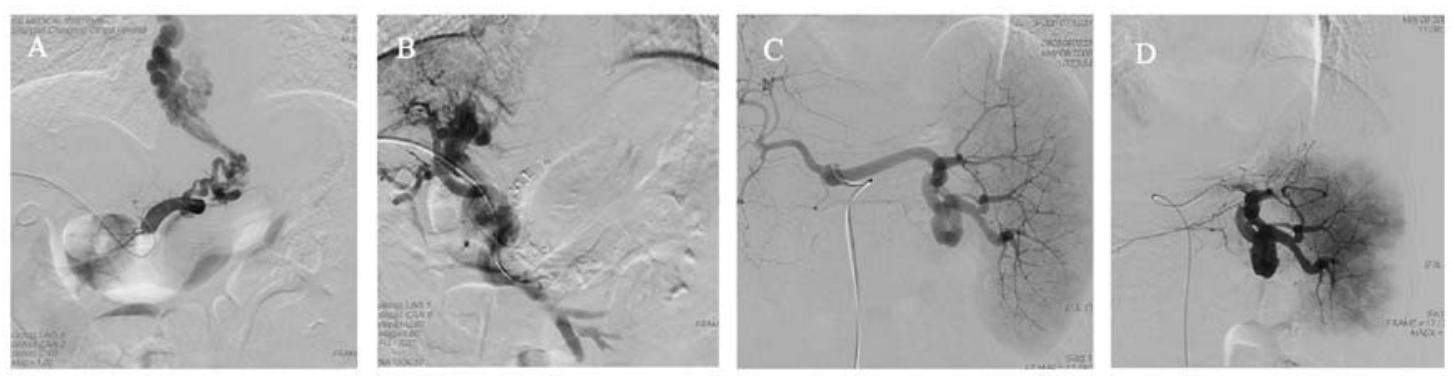

Figure 1. Esophageal varices by digital subtraction angiography (DSA) in patients before and after treatment. (A) Varicose vein shown in portal vein angiography prior to intervention. (B) Varicose vein occlusion after percutaneous transhepatic variceal embolization (PTVE) intervention. (C) Enlarged spleen shown in spleen DSA angiography prior to partial splenic embolization (PSE) intervention. (D) Partial spleen embolization shown on spleen DSA angiography after PSE intervention.
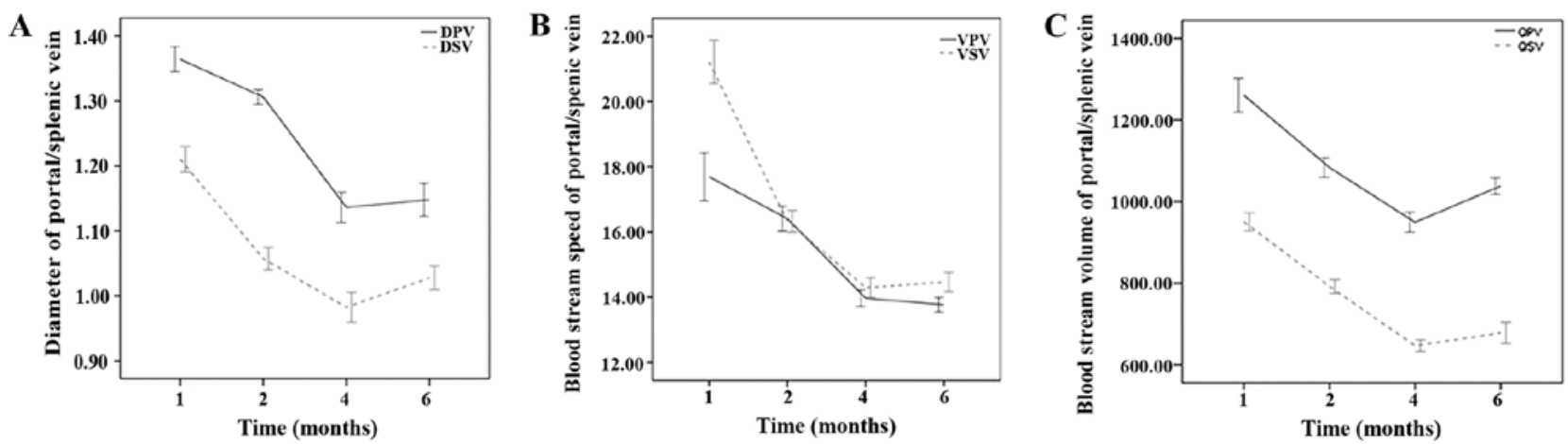

Figure 2. Hemodynamic changes in patients before and after treatment. (A) The portal/splenic vein diameters were, (B) mean blood flow velocity of portal/splenic veins and (C) QPV and QSV showed an decrease. However, these indicators were increased 6 months later, albeit not to preoperative levels. §The $\mathrm{x}$-axis represents time: 1 indicates the preoperative levels; 2 , indicates 1 month after percutaneous transhepatic variceal embolization (PTVE) + partial splenic embolization (PSE); 4 indicates 1 month after further PSE; and 6 indicates 6 months after intervention.

sequences and fragment lengths of the PCR primers. PCR was conducted under the following conditions: 40 cycles in total including initial denaturation at $94^{\circ} \mathrm{C}$ for $30 \mathrm{sec}$, denaturation at $94^{\circ} \mathrm{C}$ for $20 \mathrm{sec}$, annealing at $61^{\circ} \mathrm{C}$ for $30 \mathrm{sec}$ and extension at $72^{\circ} \mathrm{C}$ for $30 \mathrm{sec}$. After these cycles, PCR was extended at $72^{\circ} \mathrm{C}$ for $1 \mathrm{~min}$ and terminated at $4^{\circ} \mathrm{C}$.

Western blotting. To extract the protein, 100-400 $\mu \mathrm{l}$ of SDS lysis solution (containing $50 \mathrm{mM}$ Tris $\mathrm{pH} 8.1,1 \%$ SDS, and inhibitors such as sodium pyrophosphate, $\beta$-glycerophosphate, sodium orthovanadate, sodium fluoride, EDTA and leupeptin) was added to the cells in each tube. The cells were placed on ice in a high-speed electric homogenizer for $1 \mathrm{~min}$ followed by ultrasonic cracking three times within $20 \mathrm{sec}$, and centrifugation at $12,000 \mathrm{x} \mathrm{g}$ and $4^{\circ} \mathrm{C}$ for $20 \mathrm{~min}$. After centrifugation, the supernatant was transferred to a $0.5 \mathrm{ml}$ centrifuge tube and preserved at $-80^{\circ} \mathrm{C}$. The $\mathrm{BCA}$ protein assay was used for sodium dodecyl sulfate-polyacrylamide gel (SDS-PAGE) protein electrophoresis, transmembrane immune response, and development and gel image analysis of EBI3, FOXP3 and IL-17.

Statistical analysis. SPSS 13.0 software was used for statistical analysis and the main values were expressed as mean \pm SD. The enumerative data were examined using the $\chi^{2}$ test, the differences in quantitative data between groups were determined by variance analysis, and pairwise comparison was performed using the Q-test. Correlations were analyzed by the Pearson's correlations method, and all statistical tests were bilateral probability tests $(\alpha=0.05)$. $\mathrm{P}<0.05$ was considered to indicate statistical significance.

\section{Results}

Success rate of surgery. The patients successfully received the joint intervention and all 53 cases underwent emergency hemostasis resulting in an emergency hemostatic rate of $100 \%$. Bleeding stopped immediately after surgery following emergency hemostasis and varicose veins disappeared after the intervention (Fig. 1).

Hemodynamic changes. Indicators that were significantly reduced 1 month after PTVE + PSE included: portal vein diameter $(\mathrm{P}<0.01)$, blood flow $(\mathrm{P}<0.01)$, mean blood flow velocity $(\mathrm{P}<0.01)$, splenic vein diameter $(\mathrm{P}<0.01)$, blood flow of splenic vein and mean blood flow velocity of splenic vein $(\mathrm{P}<0.01)$. The portal/splenic vein diameter, portal/splenic blood flow and mean blood flow velocity 1 month after further PSE were significantly reduced, compared to these values 1 month after $\mathrm{PTVE}+\mathrm{PSE}(\mathrm{P}<0.01)$. All indicators increased 6 months later, but were much lower than preoperative levels $(\mathrm{P}<0.01)$ (Fig. 2) and showed statistically significant differences.

Compared with the preoperative levels, the following indicators were significantly reduced 1 month after PTVE + PSE: DPV $(1.30 \pm 0.04$ vs. $1.36 \pm 0.07, \mathrm{P}=0.000)$ and $\mathrm{DSV}(1.06 \pm 0.06$ vs. $1.21 \pm 0.07, \mathrm{P}=0.000)$. The portal/splenic vein diameters 1 month after further PSE were significantly reduced, compared to these 

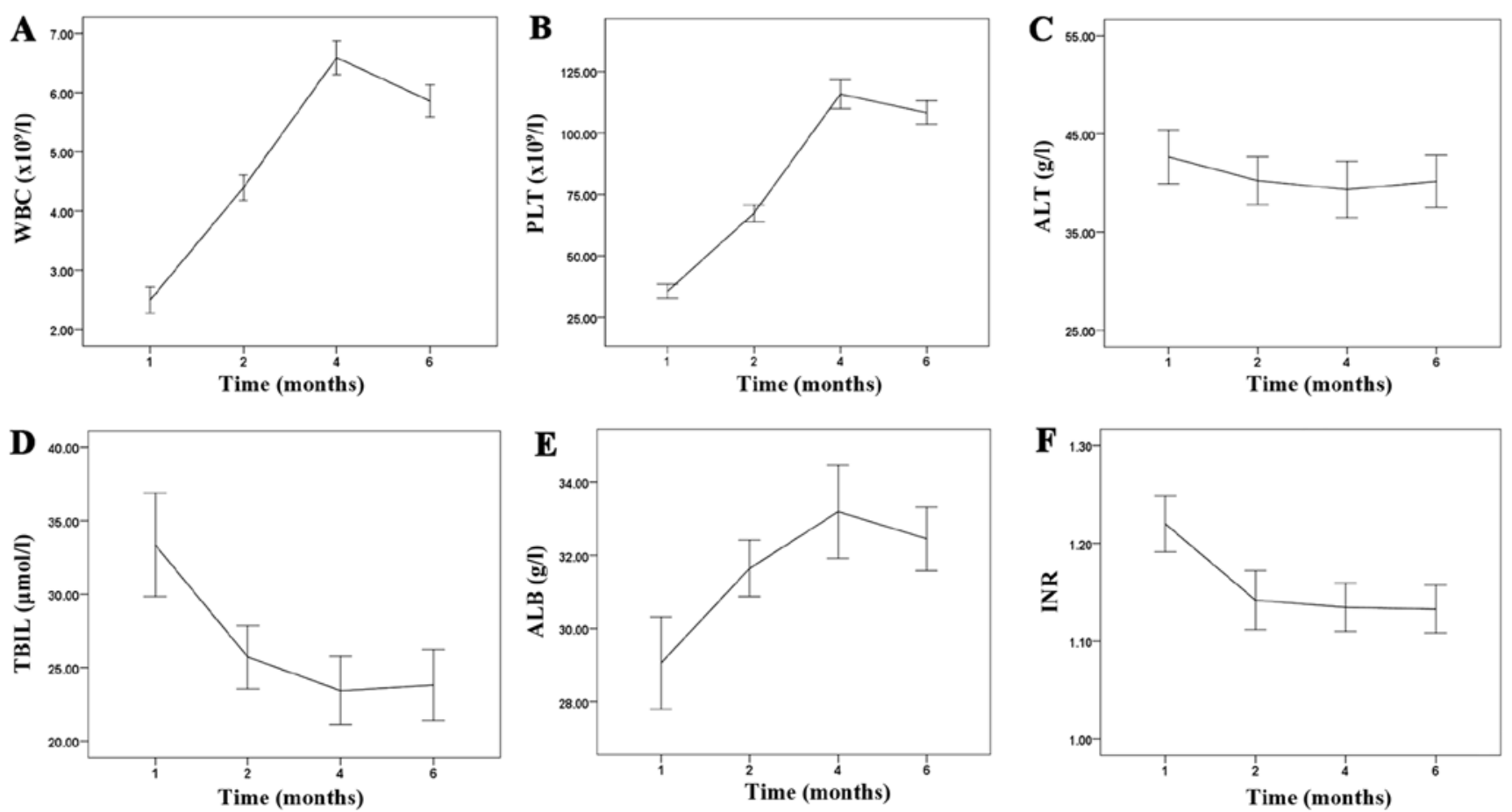

Figure 3. Changes in blood chemistry and liver function in patients before and after treatment. (A, B and E) Compared with the preoperative WBC and PLT count, a significant increase was observed 1 month after PTVE + PSE which increased further 1 month after additional PSE. The WBC and PLT counts decreased slightly 6 months later, but were significantly increased compared with the preoperative level. (C) The level of ALT did not change significantly. (D and F) Compared with the preoperative TBIL and INR levels, a significant decrease was observed 1 month after PTVE + PSE which did not decrease 1 month after further PSE. TBIL and INR increased slightly 6 months later, but were significantly decreased compared with the preoperative levels. WBC, white blood cell; PLT, platelet; TBIL, total bilirubin; ALB, albumin; INR, the prothrombin time/international normalized ratio; ALT, alanine transaminase.

parameters 1 month after PTVE + PSE (DPV 1.13 \pm 0.09 vs. $1.36 \pm 0.07, \mathrm{P}=0.000$; DSV $0.98 \pm 0.08$ vs. $1.06 \pm 0.06, \mathrm{P}=0.000)$. All indicators increased 6 months later, but were much lower than the preoperative levels (DPV $1.15 \pm 0.09$ vs. $1.36 \pm 0.07$, $\mathrm{P}=0.000$; DSV $1.03 \pm 0.07$ vs. $1.21 \pm 0.07, \mathrm{P}=0.000)$ (Fig. $2 \mathrm{~A})$

Compared with preoperative levels, the following indicators were significantly reduced 1 month after PTVE + PSE: the mean blood flow velocity of portal vein (VPV) $(16.41 \pm 1.39$ vs. $17.69 \pm 2.67, \mathrm{P}=0.000)$ and mean blood flow velocity of splenic vein (VSV) $(16.33 \pm 1.21$ vs. $21.22 \pm 2.41, \mathrm{P}=0.000)$. The mean blood flow velocity of portal/splenic veins 1 month after further PSE was significantly reduced, compared to these levels 1 month after PTVE + PSE (VPV $13.96 \pm 0.93$ vs. $16.41 \pm 1.39$, $\mathrm{P}=0.000$; VSV $14.29 \pm 1.12$ vs. $16.33 \pm 1.21, \mathrm{P}=0.000)$. All indicators increased 6 months later, but were much lower than the preoperative levels (VPV $13.77 \pm 0.81$ vs. $17.69 \pm 2.67, \mathrm{P}=0.000$; VSV $14.46 \pm 1.07$ vs. $21.22 \pm 2.41, \mathrm{P}=0.000$ ) (Fig. $2 \mathrm{~B}$ ).

Indicators that were significantly reduced 1 month after PTVE + PSE included: quantity of blood flow in the portal vein (QPV) $(1083.46 \pm 87.84$ vs. $1260.65 \pm 150.98, \mathrm{P}=0.000)$ and quantity of blood flow in the splenic vein (QSV) (792.18 \pm 60.46 vs. $950.44 \pm 80.56, \mathrm{P}=0.000$ ). The QPV and QSV 1 month after further PSE were significantly reduced, compared to the levels 1 month after PTVE + PSE (QPV 949.37 \pm 89.80 vs. $1083.46 \pm 87.84, \mathrm{P}=0.000$; QSV 646.84 \pm 5 1.88 vs. $792.18 \pm 60.46, \mathrm{P}=0.000)$. All indicators increased 6 months later, but were much lower than the preoperative levels (QPV 1038.14 \pm 74.24 vs. 1260.65 $\pm 150.98, \mathrm{P}=0.000$; QSV 678.16 \pm 94.38 vs. $950.44 \pm 80.56, \mathrm{P}=0.000$ ) (Fig. $2 \mathrm{C}$ ).
Determination of blood chemistry and liver function. Patients with obvious hypersplenism had WBC and PLT counts below the normal reference ranges prior to the intervention (Fig. 3). A gradual increase in postoperative WBC and PLT counts was observed, with marked improvement 1 and 4 months after treatment compared to the preoperative levels, respectively $(\mathrm{P}<0.01)$. WBC and PLT counts gradually decreased 6 months later, and were significantly different $(\mathrm{P}<0.01)$ compared with preoperative levels. Patients showed different degrees of liver dysfunction prior to the intervention, which gradually improved following the intervention. Postoperative ALB levels increased significantly, and TBIL and the PT of INR decreased gradually. These indicators improved each month following treatment $(\mathrm{P}<0.01)$. During the postoperative follow-up period, the level of ALT did not change significantly $(\mathrm{P}>0.05)$.

Compared with the preoperative WBC count, a significant increase was observed 1 month after PTVE + PSE (4.39 \pm 0.78 vs. $2.49 \pm 0.82, \mathrm{P}=0.000)$ and increased further 1 month after additional PSE $(6.58 \pm 1.04$ vs. $4.39 \pm 0.78, \mathrm{P}=0.000)$. The WBC count decreased slightly 6 months later, but was significantly increased compared with the preoperative level $(5.86 \pm 1.01 \mathrm{vs}$. $2.49 \pm 0.82, \mathrm{P}=0.000$ ) (Fig. 3A).

Compared with the preoperative PLT count, a significant increase was observed 1 month after PTVE + PSE $(67.37 \pm 12.21$ vs. $35.60 \pm 10.55, \mathrm{P}=0.000)$ and increased further 1 month after additional PSE $(115.86 \pm 21.09$ vs. $67.37 \pm 12.21$, $\mathrm{P}=0.000)$. The PLT count decreased slightly 6 months later, but was significantly increased compared with the preoperative level $(108.38 \pm 17.46$ vs. $35.60 \pm 10.55, \mathrm{P}=0.000)$ (Fig. 3B). 


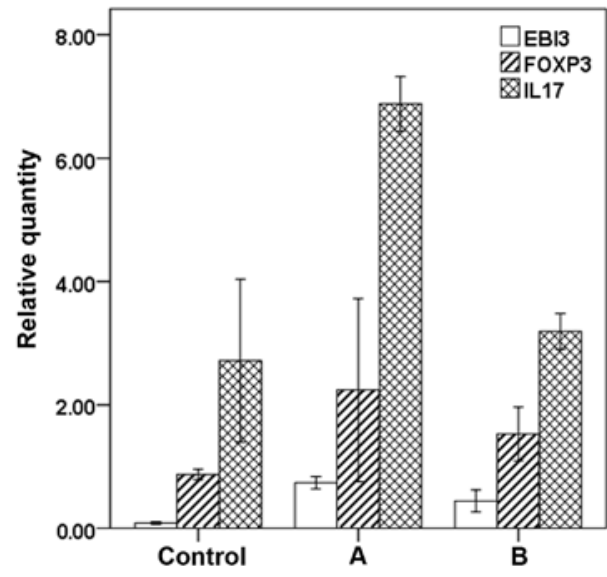

Figure 4. Fluorescent determination of the changes in expression of EBI3, FOXP3 and interleukin (IL)-17 by polymerase chain reaction (PCR) before and after treatment. Control, the normal control group; (A) the preoperative group; (B) the postoperative group.

In the postoperative follow-up period, the level of ALT did not change significantly $(\mathrm{P}>0.05)$ (Fig. 3C).

Compared with the preoperative TBIL level, a significant decrease was observed 1 month after PTVE + PSE ( $25.71 \pm 7.78$ vs. $33.35 \pm 12.81, \mathrm{P}=0.000)$, but did not decrease 1 month after further PSE (23.45 \pm 8.41 vs. $25.71 \pm 7.78$, $\mathrm{P}=0.22$ ). TBIL increased slightly 6 months later, but was significantly decreased compared with the preoperative level (23.83 \pm 8.76 vs. $33.35 \pm 12.81, \mathrm{P}=0.000)$ (Fig. $3 \mathrm{D})$.

Compared with the preoperative ALB level, a significant increase was observed 1 month after PTVE + PSE (31.64 \pm 2.81 vs. $29.05 \pm 4.59, \mathrm{P}=0.001)$ and increased slightly 1 month after further PSE (33.18 \pm 4.64 vs. 31.64 \pm 2.81 , $\mathrm{P}=0.042$ ). ALB decreased slightly 6 months later, but was significantly increased compared with the preoperative level (32.45 \pm 3.17 vs. $29.05 \pm 4.59, \mathrm{P}=0.000$ ) (Fig. $3 \mathrm{E}$ ).

Compared with the preoperative INR, a significant decrease was observed 1 month after PTVE + PSE $(1.14 \pm 0.10$ vs. $1.22 \pm 0.10, \mathrm{P}=0.000$ ), but did not decrease 1 month after further PSE ( $1.13 \pm 0.08$ vs. $1.14 \pm 0.10, P=0.70)$. INR increased slightly 6 months later, but was significantly decreased compared with the preoperative level $(1.13 \pm 0.09$ vs. $1.22 \pm 0.10$, $\mathrm{P}=0.000$ ) (Fig. 3F).

Re-bleeding rate and survival rate. During the 6-month follow-up period, no patient experienced re-bleeding or died.

Complications. Following phased joint interventional embolization, 32 patients showed different degrees of fever with body temperature ranging between 37.5 and $39.4^{\circ} \mathrm{C}$ (including 21 cases $<38.0^{\circ} \mathrm{C}$ and 11 cases $>38.0^{\circ} \mathrm{C}$ ), which was alleviated by symptomatic treatment. Twenty-nine patients experienced short-term postoperative pain and recovered following symptomatic treatment. No serious complications such as splenic rupture, peritonitis, pancreatitis, hepatic coma and ectopic embolism were noted.

Quantitative determination of EBI3, FOXP3 and IL-17 expression levels by PCR. The results of RT-qPCR showed that preoperative expression levels of EBI3 ( $\mathrm{P}<0.01)$, FOXP3

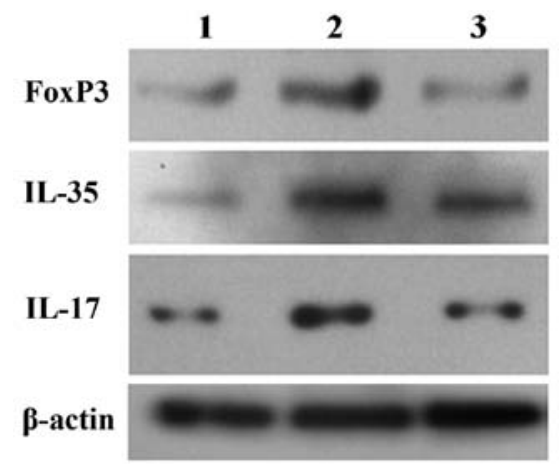

Figure 5. Changes in expression of EBI3, FOXP3 and IL-17 before and after treatment by western blotting. The expression levels of EBI3, FOXP3 and IL-17 in the postoperative group were significantly lower than the preoperative levels.

$(\mathrm{P}<0.05)$ and IL-17 $(\mathrm{P}<0.01)$ mRNA in patients were higher than those in the normal control group. Postoperative expression levels of EBI3 mRNA were higher than the normal control group $(\mathrm{P}<0.01)$. There was no significant difference $(\mathrm{P}>0.05)$ in the expression levels of FOXP3 and IL-17 between the postoperative group and normal control group. The expression levels of EBI3, FOXP3 and IL-17 mRNA in the postoperative group were significantly lower than those noted preoperatively $(\mathrm{P}<0.01)$ (Fig. 4).

The results of real-time RT-PCR showed that the expression levels of EBI3, FOXP3 and IL-17 mRNA were higher in patients undergoing phased joint interventional embolization than in the normal control group (EBI3: pre-operation: $0.74 \pm 0.09$ vs. $0.09 \pm 0.02, \mathrm{P}=0.000$; FOXP3: pre-operation: $2.24 \pm 0.74$ vs. $0.87 \pm 0.07, \mathrm{P}=0.034$ and IL-17: pre-operation: $6.88 \pm 0.38$ vs. $2.71 \pm 1.14, \mathrm{P}=0.000$ ). The expression levels of $\mathrm{EBI} 3$ mRNA were higher in patients who underwent phased joint interventional embolization, than in the normal control group $(0.44 \pm 0.15$ vs. $0.09 \pm 0.02, P=0.005)$. There were no significant differences $(\mathrm{P}>0.05)$ in the expression levels of FOXP3 and IL-17 between the postoperative group and normal control group (FOXP3: post-operation: $1.53 \pm 0.37$ vs. $0.87 \pm 0.07$, $\mathrm{P}=0.338$ and IL-17: post-operation: $3.19 \pm 0.25$ vs. $2.71 \pm 1.14$, $\mathrm{P}=0.443$ ). The expression levels of EBI3, FOXP3 and IL-17 mRNA in the postoperative group were significantly lower than preoperative levels (EBI3: $0.44 \pm 0.15$ vs. $0.74 \pm 0.09$, $\mathrm{P}=0.013$; FOXP3: $1.53 \pm 0.37$ vs. $2.24 \pm 0.74, \mathrm{P}=0.037$; IL-17: $3.19 \pm 0.25$ vs. $6.88 \pm 0.38, \mathrm{P}=0.001)$.

Determination of EBI3, FOXP3 and IL-17 expression levels using western blotting. The results of western blotting showed that the expression levels of EBI3, FOXP3 and IL-17 were higher in patients who underwent surgery than in the normal control group before and after phased joint interventional embolization. The expression levels of EBI3, FOXP3 and IL-17 in the postoperative group were significantly lower than the preoperative levels. The changes in protein expression were consistent with mRNA expression (Fig. 5).

Determination of $I L-17, I L-6$ and $I L-35$ concentrations in peripheral blood by ELISA. The results of ELISA showed that the IL-17, IL-6 and IL-35 concentrations in peripheral blood 

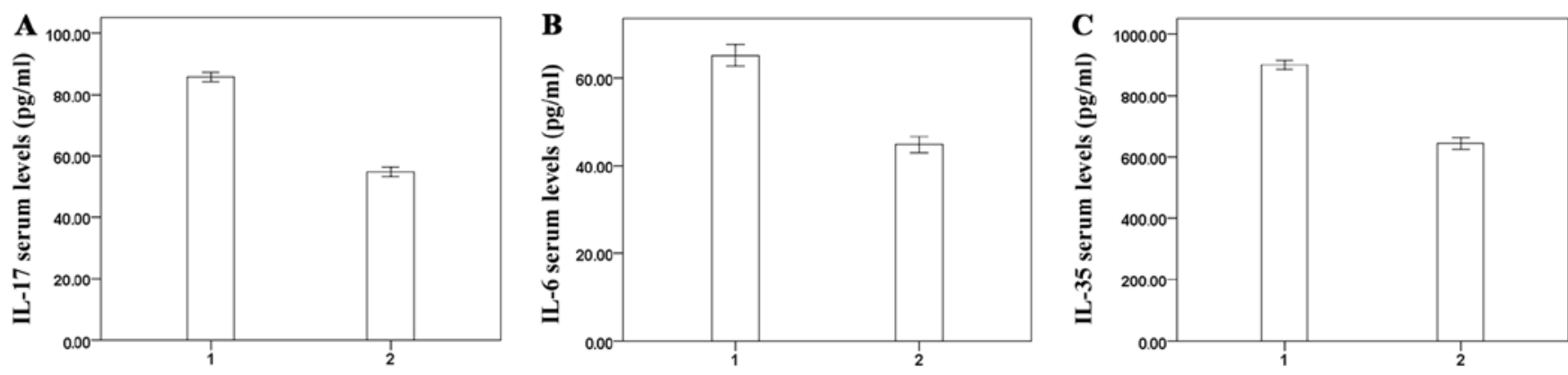

Figure 6. Changes in interleukin (IL)-35, IL-6 and IL-17 concentrations in peripheral blood by ELISA. The results showed that the IL-35, IL-6 and IL-17 concentrations in peripheral blood after phased joint intervention were reduced compared with preoperative concentrations: (A) IL-17: $85.70 \pm 5.50$ vs. 54.84 \pm 5.80 , $\mathrm{P}=0.000$; (B) IL-6: $65.16 \pm 8.98$ vs. $44.79 \pm 6.78, \mathrm{P}=0.000$ and (C) IL-35: $899.80 \pm 56.29$ vs. $643.15 \pm 68.95, \mathrm{P}=0.000$ ).

Table III. Correlation between liver function and serum concentrations of IL-35, IL-6 and IL-17.

\begin{tabular}{|c|c|c|c|c|c|c|}
\hline \multirow[b]{2}{*}{ Factors } & \multicolumn{2}{|c|}{ IL-17 } & \multicolumn{2}{|c|}{ IL-6 } & \multicolumn{2}{|c|}{ IL-35 } \\
\hline & $\mathrm{r}$ & P-value & $\mathrm{r}$ & P-value & $\mathrm{r}$ & P-value \\
\hline TBIL & 0.454 & 0.000 & 0.426 & 0.000 & 0.412 & 0.000 \\
\hline ALB & -0.369 & 0.000 & -0.264 & 0.006 & -0.372 & 0.000 \\
\hline INR & 0.331 & 0.001 & 0.293 & 0.002 & 0.320 & 0.001 \\
\hline ALT & 0.174 & 0.740 & 0.130 & 0.184 & 0.120 & 0.221 \\
\hline
\end{tabular}

IL, interleukin; TBIL, total bilirubin; ALB, albumin; INR, prothrombin time/international normalized ratio; ALT, alanine transaminase.

after phased joint intervention were significantly lower than those of the preoperative concentrations $(\mathrm{P}<0.01)$ (Fig. 6).

Correlation analysis. Correlation analysis of serum ALT, TBIL, ALB and INR with concentrations of IL-17, IL-6 and IL-35 in peripheral blood showed that serum IL-17, IL-6 and IL-35 levels were positively correlated with TBIL and INR and negatively correlated with ALB, the differences were statistically significant, ALT showed no correlations (Table III).

\section{Discussion}

PTVE mainly embolizes the gastric coronary vein and short gastric vein to block the blood flow of varicose veins and effectively stops bleeding. It is a proven method for treating portal hypertension and esophageal varices bleeding $(9,22)$. However, due to the promotion of portal vein pressure and bleeding rate using this method, its clinical applications are limited. PSE decreases the blood flow in the spleen and the return flow in the splenic vein, releases high posthepatic pressure, reduces portal blood flow and portal pressure, lowers the recurrence rate of esophageal and gastric variceal bleeding and improves symptoms of hypersplenism.

Findings of previous studies (23) have suggested that the hemogram does not return to the normal level if the splenic embolization area is $<40 \%$, while serious adverse reactions may occur if the embolization area of the splenic artery is $>80 \%$. In previous studies $(24,25)$ it has been shown that in patients with a moderately or severely enlarged spleen, at least two embolization treatments with a single range no $>50 \%$ are effective in reducing postoperative reactions and complications. Pålsson et al (26) demonstrated that the embolism area correlated with increasing PLT levels and Tajiri et al (27) observed that the significant increase in postoperative RBC and PLT counts can be maintained 7.5 and 8 years after PSE if the degree of embolism reached $\sim 70 \%$. Based on national and international research results, we conducted this phased joint intervention using a small area of PSE each time (30-40\%) and a total embolism area of $60-80 \%$, which not only ensured greater efficacy, but also minimized the incidence of complications.

This study showed that the phased joint intervention immediately controlled bleeding in 53 patients with an emergency hemostasis rate of $100 \%$, and resolved or significantly improved varices. Portal hemodynamics were improved following the phased joint intervention with portal vein diameter, splenic vein diameter, blood flow parameters, and mean blood flow velocity significantly reduced compared with the preoperative levels. These indicators increased 6 months later, but were much lower than the preoperative levels. The WBC and PLT counts increased significantly following this intervention, and were improved 1, 4 and 6 months after treatment compared with preoperative counts, respectively. Liver function after treatment was improved, with a significant increase in ALB, and decrease in TBIL and INR. These parameters remained improved 1, 4 and 6 months after treatment. The ideal range of splenic artery embolization is $60-80 \%$ without serious adverse reactions. Most patients only experience low/medium fever with short duration.

This study confirmed the satisfactory efficacy of joint interventional embolization. However, the immunological mechanisms related to the improvement in liver function remain unknown. The spleen is involved in the incidence and development of cirrhosis (28), liver cirrhosis is associated with varying degrees of splenomegaly, and the degree of splenomegaly is positively correlated with liver fibrosis in patients with chronic liver disease (29). Immune function is the most important role of the spleen, (30) in which a variety of immune cells and immune factors restrict and interact with each other. In portal hypertension and spleen enlargement, hypoxia leads to changes in the morphology and function of immune cells, and lymphocyte density is significantly reduced, although 
spleen immune function is maintained, and the total number of lymphocytes is increased when its volume increases $(31,32)$. Partial hemisection of the spleen is conducive to liver cell regeneration (33).

We suggest that phased joint embolization intervention improves hemodynamics in the liver, retains partial spleen function, and regulates the immune status thereby affecting the biological behavior of HSCs. This study has examined the effects of improved immune status following phased joint embolization on cytokine expression.

Cell immunity mainly relies on the secretion of various cytokines. Cytokines are important chemical mediators synthesized and secreted by immune cells, which act on corresponding receptors and regulate immune cell differentiation and proliferation, simultaneously, and coordinate the body's immune and inflammatory response and fibrosis progression. The role of various cytokines is not isolated, and they are synthesized and secreted through mutual adjustment. Cytokines can regulate receptor expression, affect the biological effects of the cytokine network to achieve a synergistic, antagonistic or functional expansion effect. IL-17 is mainly produced by activated Th17 cells, and as a characterized cytokine, serum IL-17 reflects the number and function of Th17 cells, to a certain extent (34-36). Sun et al (37) showed that increased Th17 cells in cirrhosis patients promoted HSC activity which led to disease progression. Additionally, Lemmers et al (38) that HSCs express IL-17R, while Wang et al (39) and Ye et al (40) found that IL-17 expression was elevated with an increase in liver fibrosis stage. This may be connected with transforming growth factor- $\beta 1$ (TGF- $\beta 1$ ) which is crucial in the development of liver fibrosis (41). The collaboration between TGF- $\beta 1$ and IL- 6 may initiate CD4+ T-cell rapid maturation of Th17 cells (42-44), which then produce IL-17. IL-6 promotes chronic liver inflammation, leading to cirrhosis.

Results of the present study have demonstrated that expression of preoperative IL-17 gene and protein was significantly increased, and the expression levels of serum IL-17 and IL-6 were significantly increased, which suggests that they are important in the development of liver fibrosis. The expression levels of IL-17 mRNA and protein were significantly decreased after the intervention, and the concentrations of IL-17 and IL-6 in peripheral blood were significantly reduced in the postoperative group, suggesting that phased joint embolization may regulate the immune status, and thus reduce IL-17, TGF- $\beta 1$ and IL-6 expression, interrupting their positive feedback loop interaction, and delaying the process of liver fibrosis.

IL-35 is a heterodimer consisting of the EB virus-induced gene 3 protein-coding (EBI3) and IL-12 p35 subunits (45) and belongs to the IL-12 family. IL-35 is secreted mainly by Treg cells, its primary physiological role is to induce the formation of Th1 cells and facilitate the proliferation of Treg cells (46). Previous studies have shown that IL-35 can inhibit the differentiation of Th17 cells and production, spleen cells from EBI3-deficient mice can produce more IL-17, and purified $\mathrm{CD} 4+\mathrm{T}$ cells from BALB/c mice cultured in medium coated with $\mathrm{CD} 3$ and $\mathrm{CD} 28$ antibody, with IL-35 added at the initial stage, resulted in significant inhibition of IL-17 generation compared with medium alone (45). The study found that TGF- $\beta$ enhanced the pro-inflammatory response by accelerating
Th17 differentiation, and IL-35 upregulated interferon- $\gamma$ (IFN- $\gamma$ ), which inhibited TGF- $\beta$ receptor downstream effector Smad-3 phosphorylation in order to block TGF- $\beta$ binding to its receptor, and prevent the differentiation of Th17 cells (36). Orphan receptor $\gamma \mathrm{t}(\mathrm{ROR} \gamma \mathrm{t})$ is an important transcription factor of Th17 cell differentiation, and EBI3 deletion increased the expression of IL-17, IL-22 and ROR $\gamma \mathrm{t}$ (47). The studies of Fontenot (48), Hori (49) and Khattri (50) demonstrated that the specific expression of FOXP3 in Treg cells is required for Treg cell development and function.

We found that mRNA and protein expression levels of IL-35, FOXP3 and IL-17 were higher than those in the normal control group before and after phased joint embolization, and serum levels of IL-17, IL-6 and IL-35 were significantly increased, which indicated that pro-inflammatory cytokines during cirrhosis can lead to pathological liver damage, while increased anti-inflammatory cytokines play a role in protecting liver cells. The mRNA expression of IL-17 in the postoperative group was significantly lower than that preoperatively, and both IL-35 and FOXP3 decreased correspondingly. Serum concentrations of expressed proteins were consistent with mRNA expression. Serum IL-35, IL-6 and IL-17 levels were positively correlated with TBIL and INR and negatively correlated with ALB, with the differences being statistically significant. No correlations were observed for ALT. ALT is an important indicator of hepatic inflammatory activation, however, ALT is susceptible to reducing enzyme drugs and other factors, and in some patients with active liver inflammation, ALT appears normal, which may be a reason for there being no correlations between serum cytokines and ALT observed in this study.

This preliminary study shows that phased joint embolization can treat esophageal varices, improve hypersplenism symptoms and liver function, effectively reduce complications, improve liver hemodynamics, retain partial spleen function which may regulate immune status, and have an impact on the expression of cytokines, which affects the biological behavior of HSCs.

This may have a significant impact on the activation and proliferation of HSCs and on expression of the extracellular matrix. The present study has shown that phased joint embolization reduced the protein and mRNA expression levels of IL-35, FOXP3 and IL-17, and inhibited the generation of IL-17, IL6, IL-35 and other cytokines, thus inhibiting the activation and proliferation of HSC, improving liver function, and delaying cirrhosis progression. As an insufficient number of cases were included in this study with a short follow-up and assessment of long-term outcome after surgery, further studies based on more cases and longer follow-up are needed.

\section{Acknowledgements}

Funding sources were received from the Shanghai Municipal Health Bureau (grant no. 2009232) and the Shanghai Municipal Health Bureau Key Disciplines (grant no. ZK2012A05).

\section{References}

1. Comar KM and Sanyal AJ: Portal hypertensive bleeding. Gastroenterol Clin North Am 32: 1079-1105, 2003. 
2. Augustin S, González A and Genescà J: Acute esophageal variceal bleeding: Current strategies and new perspectives. World J Hepatol 2: 261-274, 2010.

3. Kovalak M, Lake J, Mattek N, Eisen G, Lieberman D and Zaman A: Endoscopic screening for varices in cirrhotic patients: data from a national endoscopic database. Gastrointest Endosc 65: 82-88, 2007.

4. Groszmann RJ, Garcia-Tsao G, Bosch J, et al: Beta-blockers to prevent gastroesophageal varices in patients with cirrhosis. The N Engl J Med 353: 2254-2261, 2005.

5. Merli M, Nicolini G, Angeloni S, et al: Incidence and natural history of small esophageal varices in cirrhotic patients. J Hepatol 38: 266-272, 2003.

6. D'Amico G, Pagliaro L and Bosch J: Pharmacological treatment of portal hypertension: an evidence-based approach. Semin Liver Dis 19: 475-505, 1999.

7. Bosch J and Garcia-Pagan JC: Prevention of variceal rebleeding. Lancet 361: 952-954, 2003.

8. de Franchis R: Evolving consensus in portal hypertension. Report of the Baveno IV consensus workshop on methodology of diagnosis and therapy in portal hypertension. J Hepatol 43: 167-176, 2005.

9. Lunderquist A and Vang J: Sclerosing injection of esophageal varices through transhepatic selective catheterization of the gastric coronary vein. A preliminary report. Acta Radiol Diagn (Stockh) 15: 546-550, 1974.

10. Benner KG, Keeffe EB, Keller FS and Rösch J: Clinical outcome after percutaneous transhepatic obliteration of esophageal varices. Gastroenterology 85: 146-153, 1983.

11. Chikamori F, Kuniyoshi N, Shibuya S and Takase Y: Correlation between endoscopic and angiographic findings in patients with esophageal and isolated gastric varices. Dig Surg 18: 176-181, 2001.

12. L'Hermine C, Chastanet P, Delemazure O, Bonniere PL, Durieu JP and Paris JC: Percutaneous transhepatic embolization of gastroesophageal varices: results in 400 patients. AJR Am J Roentgenol 152: 755-760, 1989.

13. Koconis KG, Singh H and Soares G: Partial splenic embolization in the treatment of patients with portal hypertension: a review of the english language literature. J Vasc Interv Radiol 18: 463-481, 2007.

14. Shi Y, Wang Y, Shi M, et al: Clinical study of combined interventional treatment of portal hypertension. Chin J Ethnomed Ethnopharm 11: 63-65, 2010 (In Chinese).

15. Henderson NC and Iredale JP: Liver fibrosis: cellular mechanisms of progression and resolution. Clin Sci (Lond) 112: 265-280, 2007

16. Wang J, Leclercq I, Brymora JM, et al: Kupffer cells mediate leptin-induced liver fibrosis. Gastroenterology 137: 713-723, 2009.

17. Pesce JT, Ramalingam TR, Mentink-Kane MM, et al: Arginase1-expressing macrophages suppress Th2 cytokine-driven inflammation and fibrosis. PLoS Pathog 5: e1000371, 2009.

18. Gao B, Radaeva S and Park O: Liver natural killer and natural killer T cells: immunobiology and emerging roles in liver diseases. J Leukoc Biol 86: 513-528, 2009.

19. Delhem N, Carpentier A, Morales O, et al: Regulatory T-cells and hepatocellular carcinoma: implication of the regulatory $\mathrm{T}$ lymphocytes in the control of the immune response. Bull Cancer 95: 1219-1225, 2008 (In French).

20. Delhem N, Cottrez F, Carpentier A, et al: Role of the regulatory $\mathrm{T}$ lymphocytes in hepatitis $\mathrm{C}$ fibrosis progression. Bull Cancer 95 1029-1038, 2008 (In French).

21. Moriyasu F, Nishida O, Ban N, et al: Measurement of portal vascular resistance in patients with portal hypertension. Gastroenterology 90: 710-717, 1986.

22. Scott J, Dick R, Long RG and Sherlock S: Percutaneous transhepatic obliteration of gastro-oesophageal varices. Lancet 2 : 53-55, 1976.

23. Sangro B, Bilbao I, Herrero I, et al: Partial splenic embolization for the treatment of hypersplenism in cirrhosis. Hepatology 18 309-314, 1993.

24. Alwmark A, Bengmark S, Gullstrand P, Joelsson B, Lunderquist A and Owman T: Evaluation of splenic embolization in patients with portal hypertension and hypersplenism. Ann Surg 196: 518-524, 1982.

25. Moro E, Pais M, Benvegnu M, Ferrari M and Bittolo Bon G: Decrease of insulin resistance after splenectomy in a diabetic patient with liver cirrhosis and portal hypertension. Physiopathologic evaluation. Minerva Gastroenterol Dietol 40: 213-218, 1994 (In Italian).

26. Pålsson B, Hallén M, Forsberg AM and Alwmark A: Partial splenic embolization: long-term outcome. Langenbecks Arch Surg 387: 421-426, 2003.
27. Tajiri T, Onda M, Yoshida H, Mamada Y, Taniai N and Kumazaki T: Long-term hematological and biochemical effects of partial splenic embolization in hepatic cirrhosis. Hepatogastroenterology. 49: 1445-1448, 2002.

28. Yamaguchi S, Kawanaka H, Yoshida D, Maehara Y and Hashizume M: Splenic hemodynamics and decreased endothelial nitric oxide synthase in the spleen of rats with liver cirrhosis. Life Sci 80: 2036-2044, 2007.

29. Hoefs JC, Wang FW, Lilien DL, Walker B and Kanel G: A novel, simple method of functional spleen volume calculation by liverspleen scan. J Nucl Med 40: 1745-1755, 1999.

30. Chadburn A: The spleen: anatomy and anatomical function. Semin Hematol 37 (Suppl 1): S13-S21, 2000.

31. Okamoto A, Fujio K, van Rooijen N, et al: Splenic phagocytes promote responses to nucleosomes in (NZB x NZW) F1 mice. J Immunol 181: 5264-5271, 2008.

32. Sekiguchi T, Nagamine T, Takagi $\mathrm{H}$ and Mori M: Autoimmune thrombocytopenia in response to splenectomy in cirrhotic patients with accompanying hepatitis C. World J Gastroenterol 12: 1205-1210, 2006.

33. Li ZF, Zhang S, Lv GB, et al: Changes in count and function of splenic lymphocytes from patients with portal hypertension. World J Gastroenterol 14: 2377-2382, 2008

34. Harrington LE, Hatton RD, Mangan PR, et al: Interleukin 17-producing $\mathrm{CD} 4^{+}$effector $\mathrm{T}$ cells develop via a lineage distinct from the $\mathrm{T}$ helper type 1 and 2 lineages. Nat Immunol 6: 1123-1132, 2005.

35. Infante-Duarte C, Horton HF, Byrne MC and Kamradt $\mathrm{T}$ Microbial lipopeptides induce the production of IL-17 in Th cells. J Immunol 165: 6107-6115, 2000

36. Park H, Li Z, Yang XO, et al: A distinct lineage of CD4 T cells regulates tissue inflammation by producing interleukin 17 . Nat Immunol 6: 1133-1141, 2005.

37. SunHQ,Zhang JY,Zhang H,ZouZS, WangFSand JiaJH:Increased Th17 cells contribute to disease progression in patients with HBV-associated liver cirrhosis. J Viral Hepat 19: 396-403, 2012.

38. Lemmers A, Moreno C, Gustot T, et al: The interleukin-17 pathway is involved in human alcoholic liver disease. Hepatology 49: 646-657, 2009

39. Wang L, Chen S and Xu K: IL-17 expression is correlated with hepatitis B-related liver diseases and fibrosis. Int J Mol Med 27: 385-392, 2011.

40. Ye Y, Xie X, Yu J, et al: Involvement of Th17 and Th1 effector responses in patients with Hepatitis B. J Clin Immunol 30: 546-555, 2010

41. Li H, Zheng HW, Chen H, et al: Hepatitis B virus particles preferably induce Kupffer cells to produce TGF- $\beta 1$ over proinflammatory cytokines. Dig Liver Dis 44: 328-333, 2012.

42. Bettelli E, Carrier Y, Gao W, et al: Reciprocal developmental pathways for the generation of pathogenic effector TH17 and regulatory T cells. Nature 441: 235-238, 2006.

43. Mangan PR, Harrington LE, O'Quinn DB, et al: Transforming growth factor-beta induces development of the $\mathrm{T}(\mathrm{H}) 17$ lineage. Nature 441: 231-234, 2006

44. Veldhoen M, Hocking RJ, Atkins CJ, Locksley RM and Stockinger B: TGFbeta in the context of an inflammatory cytokine milieu supports de novo differentiation of IL-17producing T cells. Immunity 24: 179-189, 2006.

45. Niedbala W, Wei XQ, Cai B, et al: IL-35 is a novel cytokine with therapeutic effects against collagen-induced arthritis through the expansion of regulatory $\mathrm{T}$ cells and suppression of Th17 cells. Eur J Immunol 37: 3021-3029, 2007.

46. Collison LW, Chaturvedi V, Henderson AL, et al: IL-35mediated induction of a potent regulatory $\mathrm{T}$ cell population. Nat Immunol 11: 1093-1101, 2010.

47. Yang J, Yang M, Htut TM, et al: Epstein-Barr virus-induced gene 3 negatively regulates IL-17, IL-22 and RORgamma t. Eur J Immunol 38: 1204-1214, 2008.

48. Fontenot JD, Gavin MA and Rudensky AY: Foxp3 programs the development and function of $\mathrm{CD} 4{ }^{+} \mathrm{CD} 25^{+}$regulatory T cells. Nat Immunol 4: 330-336, 2003

49. Hori S, Nomura T and Sakaguchi S: Control of regulatory $T$ cell development by the transcription factor Foxp3. Science 299: 1057-1061, 2003

50. Khattri R, Cox T, Yasayko SA and Ramsdell F: An essential role for Scurfin in $\mathrm{CD} 4^{+} \mathrm{CD} 25^{+} \mathrm{T}$ regulatory cells. Nat Immunol 4: 337-342, 2003. 